\title{
Quantitative assessment of cross-correlation based particle velocity measurement in different gas fluidization regimes
}

\author{
Hongliang Xiao ${ }^{1}$, Yongmin Zhang ${ }^{2}$, Yiyang Hua ${ }^{1}$, and Adefarati Oloruntoba ${ }^{1}$ \\ ${ }^{1}$ China University of Petroleum Beijing \\ ${ }^{2}$ China University of Petroleum, Beijing
}

November 10, 2020

\begin{abstract}
Particle velocity measurement based on cross-correlation algorithm is explored widely in multiphase flow systems. In this study, an optical fiber probe, i.e. Labasys (B) 100 from MSE Meili was used to systematically investigate the big differences in the measurement quality of cross-correlation particle velocity instrument in fast and bubbling fluidized beds (FFB and BFB). Compared to an FFB, the measured particle velocity data in a BFB show poor data repeatability, higher percentage of invalid data and high sensitivity to the selection of the threshold value of maximum cross-correlation coefficient, corresponding to a much lower measurement quality throughout the bed. High measurement qualities appear in the annulus of the riser and under higher superficial gas velocity. A matchup relationship between the particle velocity measurement quality and the shape of the probability density function (PDF) curves of maximum cross-correlation coefficient is found. Narrower PDF curves correspond to higher measurement quality and vice versa.
\end{abstract}

\section{Hosted file}

Manuscript.pdf available at https://authorea.com/users/374661/articles/492104-quantitativeassessment-of-cross-correlation-based-particle-velocity-measurement-in-different-gasfluidization-regimes 